\title{
Application of optimal HAM for solving the fractional order logistic equation
}

\author{
Mohamed S. Mohamed ${ }^{1,2}$ \\ ${ }^{1}$ Mathematics Department, Faculty of Science, Al-Azhar University, Egypt \\ ${ }^{2}$ Mathematics Department, Faculty of Science, Taif University, Saudi Arabia
}

Email address:

m_s_mohamed2000@yahoo.com

\section{To cite this article:}

Mohamed S. Mohamed. Application of Optimal HAM for Solving the Fractional Order Logistic Equation. Applied and Computational Mathematics. Vol. 3, No.1, 2013, pp. 27-31. doi: 10.11648/j.acm.20140301.14

\begin{abstract}
In this paper, we use the optimal homotopy analysis method (OHAM) for approximate solutions of the fractional order Logistic equation. The numerical results obtained are compared with the results obtained by using variational iteration method (VIM) and Adomian decomposition method (ADM). The fractional derivatives are described by Caputo's sense. Exact and/or approximate analytical solutions of these equations are obtained. The results reveal that this method is very effective and powerful to obtain the approximate solutions.
\end{abstract}

Keywords: Logistic Equation, Fractional Order-Differential Equations, Homotopy Analysis Method, Optimal Value, Caputo's Fractional Derivative

\section{Introduction}

The subject of fractional calculus and its applications (that is, the theory of integrals and derivatives of any arbitrary real or complex order) has gained considerable popularity and importance during the past three decades or so, mainly due to its applications in diverse fields of science and engineering. Recently, the nonlinear oscillation of earthquakes can be modeled with fractional derivatives.

In recent years, fractional differential equations (FDEs) have found applications in many problems in physics and engineering [1-4]. Since most of the nonlinear FDEs cannot be solved exactly, approximate and numerical methods must be used. Some of the recent analytic methods for solving nonlinear problems include the Adomian decomposition method (ADM) [5-6], Homotopy-perturbation method (HPM) [7-8], variational iteration method (VIM) [9-10] and homotopy analysis method (HAM) [11]. The Homotopy analysis method (HAM) [12-21] is a general analytic scheme to get series solutions of various types of linear and nonlinear equations. In this work, we will implement the optimal homotopy analysis method (OHAM) to obtain the numerical solutions of the following fractional order logistic equation of the form considered in [22-25].

El-Sayed et al. [22-23] and Sweilam et al. [24-25] were concerned with the fractional-order logistic equation. They studied the stability, existence, uniqueness and numerical solution of the fractional order logistic equation.

The aim of this paper is to directly extend the (OHAM) to consider the numerical solution of the fractional order logistic equation of the form

$$
D^{\alpha} u(t)=\rho u(t)(1-u(t)), \quad t>0, \quad 0<\alpha<1, \rho>0
$$

with initial condition

$$
u(0)=x_{0}
$$

The fractional derivatives are considered in the Caputo sense. The general response expression contains a parameter describing the order of the fractional derivative that can be varied to obtain various responses. In the case of $\alpha=1$, Eq. (1.1) reduces to the classical nonlinear logistic equation.

The paper has been organized as follows. In Section 2, a brief review of the theory of fractional calculus will be given to fix notation and provide a convenient reference. In Section 3, we give analysis of the OHAM. In Section 4, we extend the application of the OHAM to construct numerical solution for the fractional order logistic equation. Conclusions are presents in section 5 . 


\section{Definitions of Fractional Derivatives and Integrals}

In these sections, we give some definitions and properties of the fractional calculus. Several definitions of fractional calculus have been proposed in the last two centuries. There are many books [1-4] that develop fractional calculus and various definitions of fractional integration and differentiation, such as Grunwald-Letnikov's definition, Riemann-Liouville definition, and Caputo's definition and generalized function approach. For the purpose of this paper, the Caputo's definition of the fractional differentiation will be used, taking the advantage of Caputo's approach that the initial conditions for fractional differential equation with Caputo's derivatives take on the traditional form as for integer-order differential equation.

Definition 2. 1. A real function $h(t), t>0$, is said to be in the space $C_{\mu}, \mu \in R$, if there exists a real number $p>\mu$, such that $h(t)=t^{p} h_{1}(t)$, where $h_{1}(t) \in C(0, \infty)$, and it is said to be in the space C ${ }_{\mu}^{n}$ if and only if $h^{(n)} \in C_{\mu}, n \in N$.

Definition 2.2. The Riemann-Liouville fractional integral operator $\left(J^{\alpha}\right)$ of order $\alpha \geq 0$, of a function $h \in C_{\mu}, \mu \geq-1$, is defined as

$$
\begin{aligned}
J^{\alpha} h(t) & =\frac{1}{\Gamma(\alpha)} \int_{0}^{t}(t-\tau)^{\alpha-1} h(\tau) d \tau \quad(\alpha>0) \\
J^{0} h(t) & =h(t)
\end{aligned}
$$

$\Gamma(\alpha)$ is the well- known Gamma function. Some of the properties of the operator $J^{\alpha}$, which we will need here, are as follows:

(1) $J^{\alpha} J^{\beta} h(t)=J^{\alpha+\beta} h(t)$,

(2) $J^{\alpha} J^{\beta} h(t)=J^{\beta} J^{\alpha} h(t)$,

(3) $J^{\alpha} t^{\gamma}=\frac{\Gamma(\gamma+1)}{\Gamma(\alpha+\gamma+1)} t^{\alpha+\gamma}$.

Definition 2. 3. The fractional derivative $\left(D^{\alpha}\right)$ of $h(t)$ in the Caputo's sense is defined as

$$
\begin{aligned}
& D^{\alpha} h(t)=\frac{1}{\Gamma(n-\alpha)} \int_{0}^{t}(t-\tau)^{n-\alpha-1} h^{(n)}(\tau) d \tau, \\
& \text { for } n-1<\alpha \leq n, \quad n \in N, t>0, h \in C_{-1}^{n} .
\end{aligned}
$$

The following are two basic properties of Caputo's fractional Derivative [4]:

(1) Let $h \in C_{-1}^{n}, n \in N$. Then $D^{\alpha} h, 0 \leq \alpha \leq n$ is well defined and $D^{\alpha} h \in C_{-1}$.

(2) Let $n-1<\alpha \leq n, n \in N$ and $h \in C_{\mu}^{n}, \mu \geq-1$. Then

$$
\left(J^{\alpha} D^{\alpha}\right) h(t)=h(t)-\sum_{k=0}^{n-1} h^{(k)}\left(0^{+}\right) \frac{t^{k}}{k !} .
$$

\section{Basic Idea of Optimal Homotopy Analysis Method (OHAM)}

For more clarifications about these basic ideas of the OHAM for nonlinear partial differential equations. It's better to see the following nonlinear partial differential equation:

$$
N[u(x, t)]=0,
$$

Where $N$ is a nonlinear operator for this problem, $x$ and $t$ denotes the independent variables, and $u(x, t)$ is an unknown function.

Through using the HAM, we first construct zero-order deformation equation

$$
\begin{aligned}
& (1-q) \ell\left[\varphi(x, t ; q)-u_{0}(x, t)\right] \\
& =q h H(t) N[\varphi(x, t, q)],
\end{aligned}
$$

Where $q \in[0,1]$ is the embedding parameter, $h \neq 0$ is an auxiliary parameter, $H(t) \neq 0$ is an auxiliary function, $\ell$ is an auxiliary linear operator, $u_{0}(x, t)$ is an initial guess, at $q=0$ and $q=1$, we have

$$
\phi(x, t ; 0)=u_{0}(x, t), \phi(x, t ; 1)=u(x, t) .
$$

By considering a Taylor series expression of $\phi(x, t, q)$ with respect to $q$ in the form

$$
\phi(x, t ; q)=u_{0}(x, t)+\sum_{m=1}^{\infty} u_{m}(x, t) q^{m}
$$

where

$$
u_{m}(x, t)=\left.\frac{1}{m !} \frac{\partial^{m} \phi(x, t ; q)}{\partial q^{m}}\right|_{q=0}
$$

the initial guess, the auxiliary function $H(t)$ and the auxiliary parameter $h$ are selected such that the series (3.4) is convergent at $q=1$, then we have from (3.4) Liao [10] and [11]

$$
u(x, t)=u_{0}(x, t)+\sum_{m=1}^{\infty} u_{m}(x, t) .
$$

We give the definition of the vector

$$
u_{n}(t)=\left\{u_{0}(x, t), u_{1}(x, t), u_{2}(x, t), \ldots . ., u_{n}(x, t)\right\} .
$$

Differentiating (3.2) $m$ times with respect to $q$, then setting $q=0$ and dividing then by $m$ ! , we have the $m^{t h}$ - order deformation equation

$$
\ell\left[u_{m}(x, t)-x_{m} u_{m-1}(x, t)\right]=h H(t) \mathbf{R}_{m}\left(u_{m-1}\right),
$$

where 


$$
\mathbf{R}_{m}\left(u_{m-1}\right)=\left.\frac{1}{(m-1) !} \frac{\partial^{m-1} \mathbf{N}[\phi(x, t ; q)]}{\partial q^{m-1}}\right|_{q=0},
$$

and

$$
x_{m}= \begin{cases}0 & m \leq 1 \\ 1 & m>1\end{cases}
$$

Applying the integral operator on both side of (3.8), to get

$$
u_{m}(x, t)=x_{m} u_{m-1}(x, t)+h \int_{0}^{t} H(t) \mathbf{R}_{m}\left(u_{m-1}\right) d t
$$

the $m^{\text {th }}$ - order deformation Eq. (3.8) is linear so we can solve it easily, especially by means of symbolic computation software such as Mathematical.

S.J. Liao [26], Yabushita et al. [27] and Mohamed S. Mohamed et al. [28, 29] they suggested the so called optimization method to find out the optimal convergence control parameters by minimum of the square residual error integrated in the whole region having physical meaning. Their method depends on the square residual error. Let $\Delta(h)$ denote the square residual error of the governing equation (3.1) and express as

$$
\Delta(h)=\int_{\Omega}(N[u(t)])^{2} d \Omega
$$

Where

$$
u_{m}(t)=u_{0}(t)+\sum_{k=1}^{m} u_{k}(t)
$$

And the optimal value of $h$ is given by solving the following a equation as:

$$
\frac{d \Delta(h)}{d h}=0
$$

\section{Solving Fractional Order Logistic Equation by the Optimal Homotopy Analysis Method (OHAM)}

In this section, to demonstrate the effectiveness of our approach, we apply the OHAM to construct approximate solutions for the nonlinear fractional order logistic equation of the form

$$
D^{\alpha} u(t)=\rho u(t)(1-u(t)), \quad t>0,0<\alpha \leq 1, \rho>0
$$

with initial condition

$$
u(0)=x_{0}
$$

The exact solution of this equation for $\alpha=1$, the ODE case, is

$$
u(t)=\frac{e^{\rho t}}{1+e^{\rho t}}
$$

Accordingly, and by the initial conditions (4.2), we can choose

$$
u_{0}(t)=u(0)=x_{0}
$$

as the initial guess of $u(t)$, and we choose the auxiliary linear operator

$$
\ell[U(t ; q)]=D^{\alpha}[U(t ; q)]
$$

with the property $\ell(C)=0$, where $\mathrm{C}$ is an integral constant.

Finally, for simplicity, we choose $H(t)=1$ as the auxiliary function. Hence, we construct the zeroth-order deformation equation

$$
(1-q) £\left(U(t ; q)-u_{0}(t)\right)=q h N(U(t ; q)),
$$

where

$$
N(U(t ; q))=D^{\alpha}(U(t ; q))-\rho U(t ; q)+\rho U^{2}(t ; q) .
$$

Differentiating (4.4) $m$ times with respect to $q$, then $q=0$ and finally dividing them by $m !$, we have the $m t h$ -order deformation equation

$$
\ell\left(u_{m}(t)-x_{m} u_{m-1}(t)\right)=h \mathbf{R}_{m}\left(u_{m-1}\right)
$$

where

$$
\mathbf{R}_{m}\left(u_{m-1}\right)=\left.\frac{1}{(m-1) !} \frac{\partial^{m-1} N(U(t ; q))}{\partial q^{m-1}}\right|_{q=0},
$$

From (4.4) and (4.7), we have

$$
\mathbf{R}_{m}\left(u_{m-1}\right)=D^{\alpha} u_{m-1}-\rho u_{m-1}+\rho \sum_{j=0}^{m-1} u_{j} u_{m-1-j}
$$

Applying the fractional integral operator, $J^{\alpha}$, to both sides of (4.6), we obtain

$$
u_{m}(t)=x_{m} u_{m-1}(t)+h J^{\alpha} \mathbf{R}_{m}\left(u_{m-1}\right), \quad m \geq 1 .
$$

The first two terms of the HAM series solution are as follows:

$$
u_{1}(t)=\rho h\left(x_{0}^{2}-x_{0}\right) \frac{t^{\alpha}}{\Gamma(\alpha+1)}
$$

$$
u_{2}(t)=(1+h) u_{1}(t)+\rho^{2} h^{2}\left(x_{0}^{2}-x_{0}\right)\left(2 x_{0}-1\right) \frac{t^{2 \alpha}}{\Gamma(2 \alpha+1)}
$$

and 


$$
\begin{aligned}
u_{3}(t)= & (1+h) u_{2}(t)+\rho^{2} h^{2}(1+h)\left(x_{0}^{2}-x_{0}\right)\left(2 x_{0}-1\right) \\
& \frac{t^{2 \alpha}}{\Gamma(2 \alpha+1)}+\rho^{3} h^{3}\left(x_{0}^{2}-x_{0}\right)\left(2 x_{0}-1\right)^{2} \\
& \frac{t^{3 \alpha}}{\Gamma(3 \alpha+1)}+\rho^{2} h^{2}\left(x_{0}^{2}-x_{0}\right) \\
& \frac{t^{3 \alpha}}{\Gamma(3 \alpha+1)} \frac{\Gamma(2 \alpha+1}{\Gamma(\alpha+1) \Gamma(\alpha+1)},
\end{aligned}
$$

Hence, the HAM series solution of the initial-value problem (1.1) and (1.2) can be given by

$$
u(t)=u_{0}(t)+u_{1}(t)+u_{2}(t)+u_{3}(t)+\ldots
$$

And so on setting $h=-1$ and $\alpha=1$; we get an accurate approximation solution in the following form:

$$
\left.u_{\text {app }}\right|_{\mathrm{ADM}}=\left.u_{\text {app }}\right|_{\mathrm{HPM}}=\left.u_{\text {app }}\right|_{\mathrm{VIM}}=\left.u_{\text {app }}\right|_{\mathrm{HAM}} .
$$

The series (4.9) contain the auxiliary parameter $h$. The results (4.9), is the same approximate solutions obtained by Sweilam et al. [24-25].

To investigate the influence of $h$ on the convergence of the solution series given by the HAM, we first plot the so-called $h$-curves of $u^{\prime}(0)$. According to the $h$-curves, it is easy to discover the valid region of $h$. We used 4 terms in evaluating the approximate solution $u(x ; t)=\sum_{i=0}^{3} u_{i}(x, t)$. Note that the solution series contains the auxiliary parameter $h$ which provides us with a simple way to adjust and control the convergence of the solution series. In general, by means of the so called $h$-curve i.e., a curve of a versus $h$. As pointed by Liao [12], the valid region of $h$ is a horizontal line segment. Therefore, it is straightforward to choose an appropriate range for $h$ which ensure the convergence of the solution series. We stretch the $h$-curve of $u^{\prime}(0)$ in Fig. 1, which shows that the solution series is convergent when $-2<h<0$.

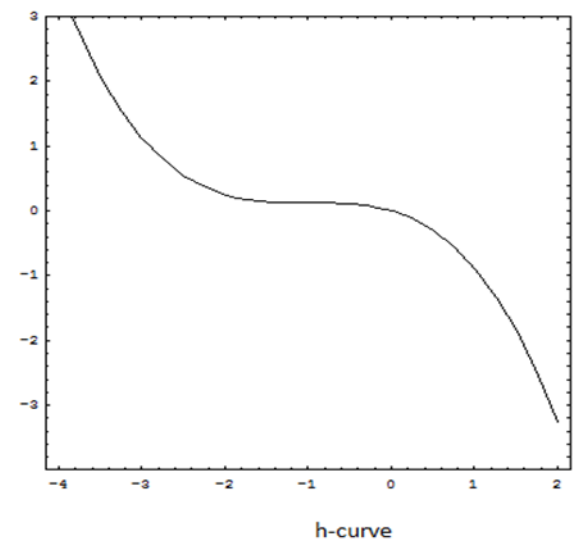

Fig 1. The $h$-curve of the three order approximate solutions (4.9) when $t=0, \alpha=1$.
As mentioned in section 3, the optimal value of $h$ is determined by minimum of $\Delta_{4}$, corresponding to the nonlinear algebraic equation $\frac{d \Delta_{4}}{d h}=0$, our calculations showed that, $d \Delta_{4}$ has its minimum values at $h=-0.834348$

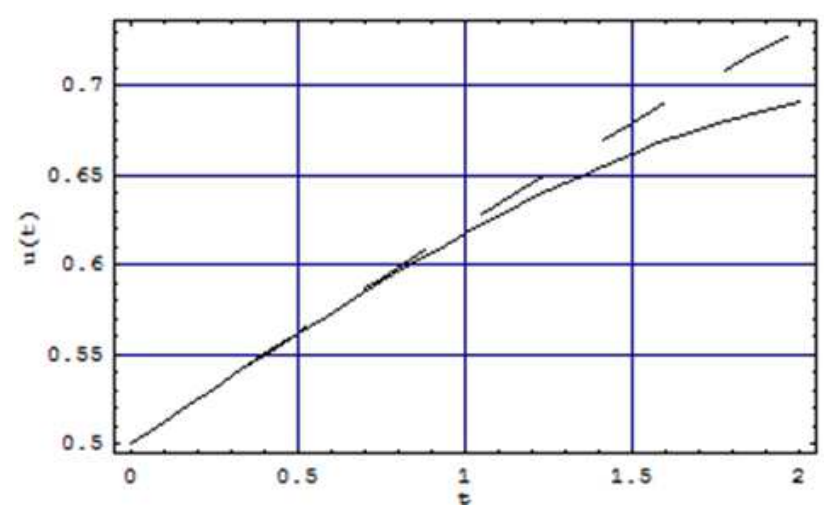

Fig 2. The exact solution (4.1) Dashing line (--- ---) is compared with the approximate solution (4.9) (-------) when $h=-0.834348$, $\rho=x_{0}=0.5$ and $\alpha=1$.

\section{Conclusion}

In this work, the OHAM was applied to derive exact and approximate analytical solutions of nonlinear fractional initial-value problems FIVPs. So, the OHAM can be applied to solve linear/nonlinear fractional differential equation. In conclusion, OHAM provides accurate numerical solution for nonlinear problems in comparison with other methods. This scheme provides us a simple way to adjust and control the convergence of the series solution by choosing proper values of auxiliary and homotopy parameters. In conclusion, HAM gives accurate approximate solution for nonlinear problems in comparison with other methods.. Mathematical has been used for computations in this paper.

\section{References}

[1] K. B. Oldham, J. Spanier, The fractional calculus, Academic press, New York 1974.

[2] K. S. Miller, B. Ross, An introduction to the fractional and fractional differential equations, John Wiley and Sons, New York, 1993.

[3] Y. Luchko, R. Gorenflo, The initial-value problem for some fractional differential equations with Caputo derivative, Preprint Series A08-98, Fachbereich Mathematik and Informatic, Freie Universitat,Berlin, 1998.

[4] I. Podlubny, Fractional differential equations, Academic press, New York, 1999.

[5] S S Ray, RK Bera, An approximate solution of a nonlinear fractional differential equation by Adomian decomposition method, Appl. Math. Comput.(167)(2005)561-571.

[6] O. Abdulaziz, I. Hashim, Chowdhury MSH, Zulkifle Ak, Assessment of decomposition method for linear and 
nonlinear fractional differential equations. Far East J Appl. Math. 28(1)(2007)95-112.

[7] S. Momani, Z. Odibat, Homotopy perturbation method for nonlinear partial differential equations of fractional order. Phys. Lett. A365 (2007)345-350.

[8] Z. Odibat, S. Momani, Modified homotopy perturbation method: application to quadratic Riccati differential equation of fractional order, Chaos Solitions Fractals. 36(2008)167-174.

[9] Z. Odibat, S. Momani, Application of variational iteration method to nonlinear differential equation of fractional order. Int J Nonlinear Sci. Num. Simul. 1(7)(2006)271-279.

[10] S. Momani, Z. Odibat. Numerical comparison of methods for solving linear differential equations of fractional order. Chaos Solitons Fractals.31(2007)1248-1255.

[11] SJ Liao, The proposed homotopy analysis technique for the solution of nonlinear problem. Ph.D thesis, Shanghai Jiao Tong University;1992.

[12] SJ Liao, An approximate solution technique which does not depend upon small parameters: a special example. Int. J. Nonlinear Mech. 30(1995)371-380.

[13] SJ Liao, An approximate solution technique which does not depend upon small parameters (II): an application in fluid mechanics, Int. J. Nonlinear Mech. 32(1997)815-822.

[14] SJ Liao, An explicit, totally analytic approximation of Blasius viscous flow problems, Int. J. Nonlinear Mech. 34(4)(1999)759-778.

[15] SJ Liao, Beyond perturbation: introduction to the homotopy analysis method,CRC Press, Boca Raton: Chapman\& Hall, 2003.

[16] SJ Liao, Notes on the homotopy analysis method: Some defintions and theorems, Commun Nonlinear Sci Numer Simulat. 14(2009)983-997.

[17] K. Hemida, M. S. Mohamed, Numerical simulation of the generalized Huxley equation by homotopy analysis method, Journal of applied functional analysis, 5(4)(2010)344-350.

[18] M. S. Mohamed, Application of homotopy analysis method to fractional order generalized Huxley equation, Journal of applied functional analysis, 7(4)(2012), pp. 367-373.

[19] M. S. Mohamed, H. Ghany, Analytic approximations for fractional-order hyperchaotic system, Journal of advanced research in Dynamical and Control Systems, 3(2011), pp. $1-12$

[20] H. A. Ghany and M. S. Mohamed, White noise functional solutions for the wick-type stochastic fractional Kdv-Burgers-Kuramoto equations, Journal of the Chinese Journal of Physics, 50(4)(2012), pp. 619-626.

[21] K. A. Gepreel and M. S. Mohamed, Analytical approximate solution for nonlinear space-time fractional Klein Gordon equation, Chinese physics B, 22(1)(2013), pp. 010201-6.

[22] A.M.A. El-Sayed, E.M. El-Mesiry and H.A.A. El- Saka, On the fractional-order logistic equation, Applied Mathematics-Letters 20(2007)817-823.

[23] A. M. A. El-Sayed, H. A. A. El-Saka, and E. M. El-Maghrabi, "On the fractional-order logistic equationwith two different delays," Zeitschrift fur Naturforschung. Section A, vol. 66, no. 3-4, pp. 223--227, 2011.

[24] N. H. Sweilam, M. M. Khader, A. M. S. Mahdy, Numerical studies for solving fractional order logistic equation,International Journal of Pure and Applied Mathematics , 78( 8) 2012, 1199-1210

[25] N. H. Sweilam, M. M. Khader, and A. M. S. Mahdy, Numerical Studies for Fractional-Order Logistic Differential Equation with Two Different Delays, Journal of Applied Mathematics, Volume 2012, Article ID 764894, 14 pages

[26] S J Liao. An optimal homotopy analysis approach for strongly nonlinear differential equations, Commun. Nonlinear Science and Numerical Simulation, 15(8)(2010) 2003-2016.

[27] K. Yabushita , M. Yamashita and K. Tsuboi. An analytic solution of projectile motion with the quadratic resistance law using the homotopy analysis method. J. Phys. A. Math.Gen., 40(2007) 8403.

[28] S. M. Abo-Dahab, M. S. Mohamed and T. A. Nofal. A One Step Optimal Homotopy Analysis Method for propagation of harmonic waves in nonlinear generalized magneto-thermo elasticity with two relaxation times under influence of rotation. Journal of in Abstract and Applied Analysis, (2013) $1-14$.

[29] K. A. Gepreel, and M. S. Mohamed. An optimal homotopy analysis method nonlinear fractional differential equation. Journal of Advanced Research in Dynamical and Control Systems, 6(2014)1-10. 